Theme: Fracture Mechanics and Structural Integrity

\title{
ON THE USE OF PRESSURE-SENSITIVE CRITERIA TO PREDICT YIELDING AND MULTIAXIAL FATIGUE DAMAGE IN METALLIC STRUCTURES*
}

\author{
Marco Antonio Meggiolaro ${ }^{1}$ \\ Jaime Tupiassú Pinho de Castro ${ }^{1}$
}

\begin{abstract}
Most cyclic plasticity models used in multiaxial fatigue life predictions are based on a concept of a yield surface, which divides the elastic and plastic domains. Plastic straining occurs when the stress state reaches the surface and tries to move outward. Subsequent plastic straining causes the stress state to remain on the yield surface, which will translate to prevent the stress point from moving outside it according to a kinematic hardening rule that models the Bauschinger effect. The accurate modeling of the yield surface shape is fundamental to predict stress-strain relations. In this work, the main yield surface equations for isotropic materials are compared. Even though most materials can be modeled using Mises and Tresca surfaces, other need to consider the effect on the yield strength of the hydrostatic stress or the normal stress acting on a critical plane. It is shown that pressuresensitive models originally devised for concrete can be applied to predict yielding in some materials, and even fatigue life for metallic structures with short cracks.
\end{abstract}

Keywords: Multiaxial fatigue; Yield surface; Pressure-sensitive materials.

1 Ph.D., Professor of Mechanical Engineering, Pontifical Catholic University of Rio de Janeiro, Rio de Janeiro, RJ, Brazil.

* Technical contribution to the $69^{\text {th }}$ ABM International Annual Congress and to the ENEMET, July $21^{\text {st }}-25^{\text {th }}$, 2014, São Paulo, SP, Brazil. 


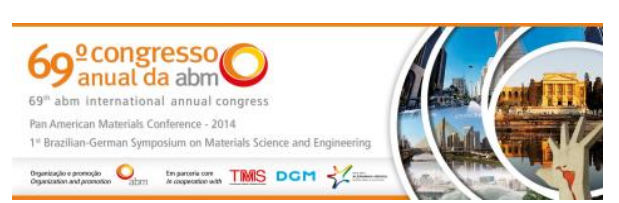

\section{INTRODUCTION}

Except for a few counter-examples [1], most plasticity models are based on a concept of a yield surface, which divides the elastic and plastic domains. When represented in the $6 D$ stress space, the yield surface is a usually convex fivedimensional (5D) surface, whose interior describes all possible combinations of the six stress components that can be assumed purely elastic. Plastic straining occurs when the stress state reaches the surface and tries to move outward. Subsequent plastic straining causes the stress state to remain on the yield surface, which will translate or change its size or shape accordingly, to prevent the stress point from moving outside it. These translations and size changes can be described by kinematic and isotropic hardening models.

The normality rule assumes that plastic straining always occurs in a direction perpendicular to the yield surface at the current stress point. The direction of the plastic flow is then described by a normal vector $\overrightarrow{\mathbf{n}}$ calculated from the gradient of the equation that defines the yield surface. Since in this case the same equation is used to define both the yield criterion and the plastic flow direction, this yield surface is said to have an associated plastic flow rule. On the other hand, if the plastic flow direction is independent of the yield surface equation, then it is said to have a nonassociated flow rule.

The yield surface equation can also be used as a static failure criterion, in the same way that the same Mises equivalent stress can be used to predict yielding, through $\sigma$ Mises $=S_{Y}$, where $S_{Y}$ is the yield strength. It can also be used as part of fatigue criteria that use equivalent stress or strain ranges $\Delta \sigma$ Mises or $\Delta \varepsilon$ Mises, as well as

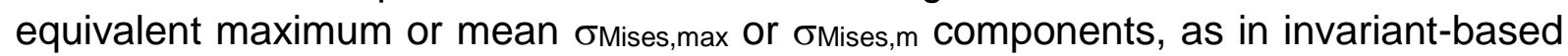
multiaxial fatigue damage models such as Sines and Crossland. The main yield surface models are described next.

\section{PRESSURE-INSENSITIVE YIELD SURFACES}

The Mises or maximum distortion energy and the Tresca or maximum shear stress yield surfaces are the most widely used for describing the yielding behavior of isotropic, pressure-insensitive, and non-SD materials, i.e. isotropic materials whose yielding is independent of the hydrostatic stress $\sigma_{\mathrm{h}}$ and whose strength difference (SD) between tension and compression is negligible.

\subsection{Mises}

The Mises criterion assumes that yielding occurs when the distortional energy of a multiaxial stress state equals or exceeds the corresponding energy associated with the uniaxial yield strength. Its resulting yield surface equation is then

$$
\sigma_{\text {Mises }}=\mathbf{S} \Rightarrow \sigma_{x}^{2}+\sigma_{y}^{2}+\sigma_{z}^{2}-\sigma_{x} \sigma_{y}-\sigma_{x} \sigma_{z}-\sigma_{y} \sigma_{z}+3 \cdot\left(\tau_{x y}^{2}+\tau_{y z}^{2}+\tau_{z x}^{2}\right)=S^{2}
$$

where $S$ is the current yield strength, e.g. the monotonic $S=S Y$ or the cyclic $S=S_{Y c}$. Isotropic strain-hardening effects, associated with a uniform expansion (or contraction) of the yield surface, can be included in the omises equation by changing the value of $S$ as the material deforms plastically. Kinematic strain-hardening, associated with plastic memory induced by the Bauschinger effect, can be modeled

\footnotetext{
* Technical contribution to the $69^{\text {th }}$ ABM International Annual Congress and to the ENEMET, July $21^{\text {st }}-25^{\text {th }}$, 2014, São Paulo, SP, Brazil.
} 
by translations of the yield surface. When represented in the principal stress coordinates $\sigma_{1}-\sigma_{2}-\sigma_{3}$, the Mises yield surface becomes

$$
\left(\sigma_{1}-\sigma_{2}\right)^{2}+\left(\sigma_{2}-\sigma_{3}\right)^{2}+\left(\sigma_{1}-\sigma_{3}\right)^{2}=2 \cdot S^{2}
$$

This equation describes the surface of an infinite cylinder with radius $S \cdot \sqrt{\mathbf{2} / \mathbf{3}}$ around the hydrostatic axis.

Since the radii $\tau_{12}=0.5 \cdot\left|\sigma_{1}-\sigma_{2}\right|, \tau_{23}=0.5 \cdot\left|\sigma_{2}-\sigma_{3}\right|$ and $\tau_{13}=0.5 \cdot\left|\sigma_{1}-\sigma_{3}\right|$ of the three Mohr circles are the principal shear stresses, the Mises theory can be interpreted as a three-shear concept that describes the yield surface as a sphere with radius $S / \sqrt{2}$ in the $\tau_{12}-\tau_{23}-\tau_{13}$ principal shear stress space, since

$$
\tau_{12}^{2}+\tau_{23}^{2}+\tau_{13}^{2}=S^{2} / 2
$$

When represented in a $\sigma_{x}-\sigma_{y}$ diagram assuming $\sigma_{z}=\tau_{x y}=\tau_{x z}=\tau_{y z}=0$, a sub-space of the 6D stress space, the Mises surface results in the boundary of an ellipse rotated $45^{\circ}$ from the $x$ axis, see Figure 1. If the $6 D$ deviatoric stress space is used, the Mises surface results in a $5 \mathrm{D}$ hypersphere with radius $S \cdot \sqrt{\mathbf{2} / \mathbf{3}}$, which simplifies to a circle in the $S_{x}-S_{y}$ deviatoric sub-space assuming $S_{z}=\tau_{x y}=\tau_{x z}=\tau_{y z}=0$, see Figure 1. Finally, in the 5D deviatoric stress space defined in [2], the Mises surface results in a 5D hypersphere with radius $S$ described by the equation $\left|\overrightarrow{\mathbf{s}}^{\prime}\right|=\sigma_{\text {Mises }}=\mathbf{S}$, which can be represented as a circle in the $\mathrm{s}_{1}$ - $\mathrm{s}_{2}$ sub-space assuming $\mathrm{s}_{3}=\mathrm{s}_{4}=\mathrm{s}_{5}=0$ (Figure 1).

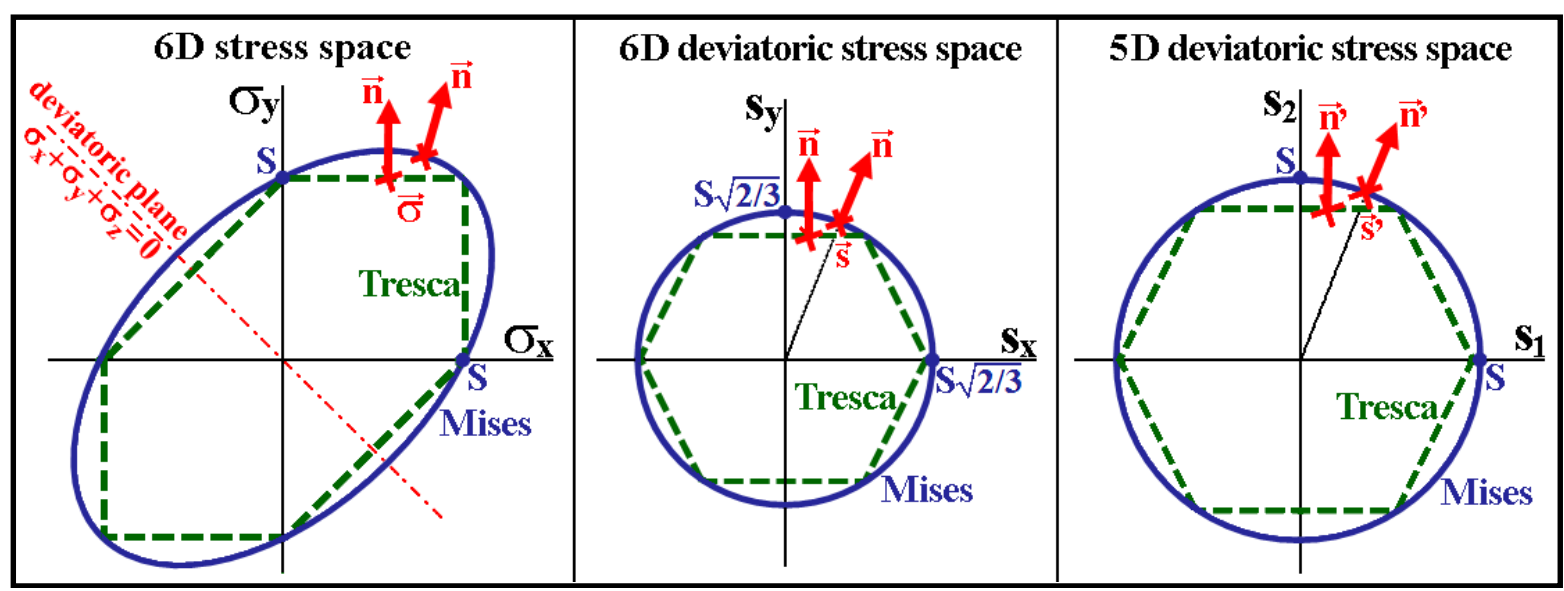

Figure 1. Cross sections of the Mises and Tresca yield surfaces on the $\sigma_{z}=0$ plane of the $6 \mathrm{D}$ stress space (left) and on the deviatoric plane $\sigma_{x}+\sigma_{y}+\sigma_{z}=0$ of the 6D deviatoric stress space (middle) and of the 5D deviatoric stress space (right), when all shear components are zero.

\subsection{Tresca}

Contrary to Mises' three-shear concept, the Tresca yield surface uses a single-shear formulation that searches for the maximum principal shear stress, given by

$$
\max \left\{\left|\sigma_{1}-\sigma_{2}\right|,\left|\sigma_{2}-\sigma_{3}\right|,\left|\sigma_{1}-\sigma_{3}\right|\right\}=S \text { or } \max \{\tau\}=\max \left\{\tau_{12}, \tau_{23}, \tau_{13}\right\}=S / 2
$$

\footnotetext{
* Technical contribution to the $69^{\text {th }} A B M$ International Annual Congress and to the ENEMET, July $21^{\text {st }}-25^{\text {th }}$, 2014, São Paulo, SP, Brazil.
} 


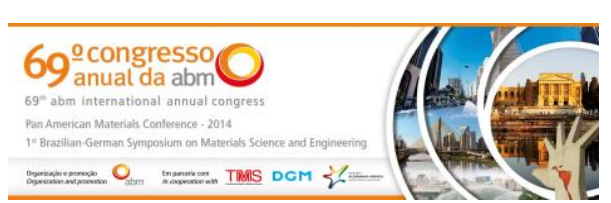

In the principal stress coordinates $\sigma_{1}-\sigma_{2}-\sigma_{3}$, the Tresca surface describes an infinite hexagonal prism inscribed inside the cylindrical Mises surface. The cross sections of the Tresca surface on the $\sigma_{z}=0$ and $\sigma_{x}+\sigma_{y}+\sigma_{z}=0$ planes are shown in Figure 1, described by a stretched hexagon in the stress space and regular hexagons in the deviatoric spaces, all of them inscribed inside the respective Mises surfaces. Figure 1 also shows the normal vectors $\overrightarrow{\mathbf{n}}$ (in 6D) or $\overrightarrow{\mathbf{n}}^{\prime}$ (in 5D) associated with the Mises and Tresca surfaces, which can be significantly different even for stress states $|\overrightarrow{\boldsymbol{\sigma}}|,|\overrightarrow{\mathbf{s}}|$ or $\left|\overrightarrow{\mathbf{S}}^{\prime}\right|$ very close to each other.

Since the normal vector is not defined at Tresca's surface corners, its original equation cannot determine plastic flow direction. A possible option is to use a nonassociated plastic flow calculated from a smooth yield surface that approximates Tresca's, for instance the Mises surface. Tresca would then be used as the yield criterion, while the Mises surface normal nearest to the current stress state would determine the plastic straining direction. Such non-associated algorithm mixing Tresca and Mises is inconvenient and rarely adopted. A much simpler approach is to consider an associated algorithm that uses Mises in both calculations.

\section{PRESSURE-SENSITIVE YIELD SURFACES}

Mises and Tresca criteria do not depend on the hydrostatic stress, therefore they cannot be used to model pressure-sensitive materials such as cast irons, whose yielding and rupture is influenced by microvoids and microcracks near graphite inclusions in their inhomogeneous microstructures. The Mises and Tresca surfaces are also symmetrical with respect to tension and compression, thus they cannot describe the behavior of SD materials, those which present a significant strength difference (SD) in tension and in compression, such as concrete, rock, soil, and other cohesive-frictional granular materials. These SD geomaterials may present SD ratios $\lambda_{S D}$ between tension and compression strengths as low as 1/15 for the ultimate strength. The material constant $\lambda \mathrm{SD}$ may be defined for either the yield or the ultimate strength ratio between tension and compression, depending whether the studied problem involves a yield or failure analysis. Therefore, yield criteria that explicitly consider this strength difference are necessary when it is desired to apply plasticity theory to such SD materials.

\subsection{Mohr-Coulomb and Rankine}

The Mohr-Coulomb criterion based on Coulomb's 1773 friction equation indirectly accounts for short cracks, voids, or even particle interface effects in inhomogeneous, fissured, or granular materials such as concrete and soil, which can affect both their yielding and rupture behavior. A tensile normal stress $\sigma_{\perp}>0$ perpendicular to the faces of such defects tends to open them, sinergically interacting with the shear stress $\tau$ during yielding or fracture. On the other hand, a compressive normal stress $\sigma_{\perp}<0$ perpendicular to their faces tends to close and to increase the friction between them, helping their shear stress resistance, see Figure 2.

For compressive normal stresses $\sigma_{\perp}<0$ perpendicular to the faces of such particles or crack-like defects, the Mohr-Coulomb criterion assumes that the friction due to the shear stress between their interfaces is equal to $-\sigma_{\perp} \cdot \tan (\phi S D)$, where $\phi S D$ is the angle of internal friction, a material constant used to describe the friction coefficient $\tan (\phi S \mathrm{SD})$ between them. Typical values of $\phi s D$ for geomaterials range from $45^{\circ}$ for dense sand

\footnotetext{
* Technical contribution to the $69^{\text {th }}$ ABM International Annual Congress and to the ENEMET, July $21^{\text {st }}-25^{\text {th }}$, 2014, São Paulo, SP, Brazil.
} 


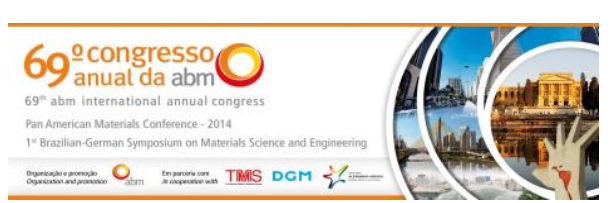

plane associated with the largest Mohr circle, which results in plane 1-3 if the principal stresses are ordered such that $\sigma_{3} \leq \sigma_{2} \leq \sigma_{1}$, at the tangency point with the Mohr-Coulomb failure line. Thus, the maximum value of $\left(\sigma_{\perp}, \tau\right)$ occurs in a direction that makes an angle $\phi s D / 2$ with respect to the maximum shear direction, which corresponds to an angle $\phi \mathrm{SD}$ (twice the direction angle) in the largest Mohr circle, see Figure 2. This solution $\left(\sigma_{\perp}, \tau\right)$ is then replaced in Equation (5), giving

$$
\left(\sigma_{\perp}, \tau\right)=\left(\sigma_{\perp 13}+\tau_{13} \sin \phi_{\mathrm{SD}}, \tau_{13} \cos \phi_{\mathrm{SD}}\right) \Rightarrow \tau_{13}+\sigma_{\perp 13} \sin \phi_{\mathrm{SD}}=\mathrm{c}_{\mathrm{SD}} \cos \phi_{\mathrm{SD}}
$$

where $\tau_{i j} \equiv\left|\sigma_{i}-\sigma_{j}\right| / 2$ are the principal shear stresses and $\sigma_{\perp i j} \equiv\left(\sigma_{\mathbf{i}}+\sigma_{\mathbf{j}}\right) / 2$ are the corresponding normal stresses, with $1 \leq \mathrm{i}<\mathrm{j} \leq 3$, see Figure 2 .

This equation can be written in a general form that does not need to assume the sorting order $\sigma_{3} \leq \sigma_{2} \leq \sigma_{1}$ through

$$
\max \left\{\tau_{12}+\alpha_{S D} \cdot \sigma_{\perp 12}, \tau_{23}+\alpha_{S D} \cdot \sigma_{\perp 23}, \tau_{13}+\alpha_{S D} \cdot \sigma_{\perp 13}\right\}=\beta_{S D}
$$

where $\alpha S D \equiv \sin (\phi S D)$ is a material constant that calibrates the influence of the normal stress on the shear strength, and $\beta \mathrm{SD} \equiv \mathrm{CSD} \cdot \cos (\phi \mathrm{SD})$ is an effective shear strength. Mohr-Coulomb's surface describes an infinite hexagonal pyramid in the principal stress coordinates $\sigma_{1}-\sigma_{2}-\sigma_{3}$, with vertex at the hydrostatic tensile state $\sigma_{x}=\sigma_{y}=\sigma_{z}$ $=\beta \mathrm{sD} / \alpha \mathrm{SD}$ and symmetry axis at the hydrostatic axis.

One limit case of Mohr-Coulomb's criterion is for a non-SD material with $\lambda S D=1$. This limit reproduces Tresca's model, since using $\alpha S D=0, \beta S D=C S D=S / 2$ and $\phi S D=0^{\circ}$ in Mohr-Coulomb's criterion results in $\max \{\tau\}=\max \left\{\tau_{12}, \tau_{23}, \tau_{13}\right\}=S / 2$. Note that the direction that maximizes the cohesive shear stress is only equal to the maximum shear stress direction when $\phi S \mathrm{SD}=0^{\circ}$, i.e. for the Tresca model.

The other limit case is for SD materials with strengths much higher in compression than in tension, i.e. $\lambda S D \cong 0 \Rightarrow \alpha S D=1, \beta S D=S, C S D \rightarrow \infty$, and $\phi S D=90^{\circ}$. Since $\alpha S D=1$ results in a failure criterion given by

$$
\tau_{\mathrm{ij}}+\alpha_{\mathrm{SD}} \cdot \sigma_{\perp \mathrm{ij}}=\left|\sigma_{\mathrm{i}}-\sigma_{\mathrm{j}}\right| / 2+\left(\sigma_{\mathrm{i}}+\sigma_{\mathrm{j}}\right) / 2=\max \left\{\sigma_{\mathrm{i}}, \sigma_{\mathrm{j}}\right\}=\mathbf{c}_{\mathrm{SD}}
$$

and since $-\max \left\{-\sigma_{\mathbf{i}},-\sigma_{\mathbf{j}}\right\}=\min \left\{\sigma_{\mathbf{i}}, \sigma_{\mathbf{j}}\right\}$, then Mohr-Coulomb's model results in the maximum principal stress or Rankine's criterion, which predicts that failure occurs when any of the principal stresses reach the material strengths in tension $S$ or in compression $-\mathrm{S} / \lambda \mathrm{sD}$, i.e.

$$
\max \left\{\sigma_{1}, \sigma_{2}, \sigma_{3}\right\}=S \text { or } \min \left\{\sigma_{1}, \sigma_{2}, \sigma_{3}\right\}=-\mathrm{S} / \lambda_{\mathrm{SD}}
$$

Rankine's maximum normal stress surface describes a cube in the principal stress coordinates $\sigma_{1}-\sigma_{2}-\sigma_{3}$. It has been satisfactorily applied as a failure criterion to brittle materials with several $\lambda \mathrm{sD}$ ratios.

Figure 3(right) shows cross sections on the tensile deviatoric plane $\sigma_{x}+\sigma_{y}+\sigma_{z}=S$ of Mohr-Coulomb surfaces $\lambda \mathrm{SD}=\{0,0.2,0.5,1\}$. The Tresca and Rankine limit cases are also shown in Figure 3(left), for $a \sigma_{z}=0$ cross section. Mohr-Coulomb's deviatoric cross sections are equilateral hexagons which become as well equiangular for the

\footnotetext{
* Technical contribution to the $69^{\text {th }}$ ABM International Annual Congress and to the ENEMET, July $21^{\text {st }}-25^{\text {th }}$, 2014, São Paulo, SP, Brazil.
} 


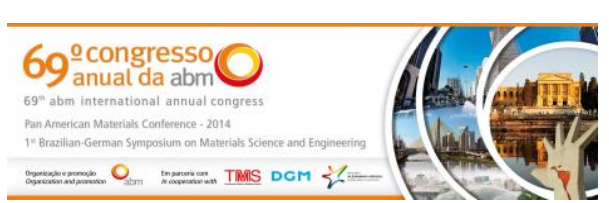

Tresca limit case $\lambda \mathrm{SD}=1$. Note that Mohr-Coulomb and Rankine only coincide if $\lambda \mathrm{SD}=$ 0 . Although Mohr-Coulomb is usually applied for $0 \leq \lambda \mathrm{SD} \leq 1$, its equation can be used as well for materials with $\lambda \mathrm{SD}>1$, such as fiber-reinforced composites with low compression strengths due to fiber buckling.

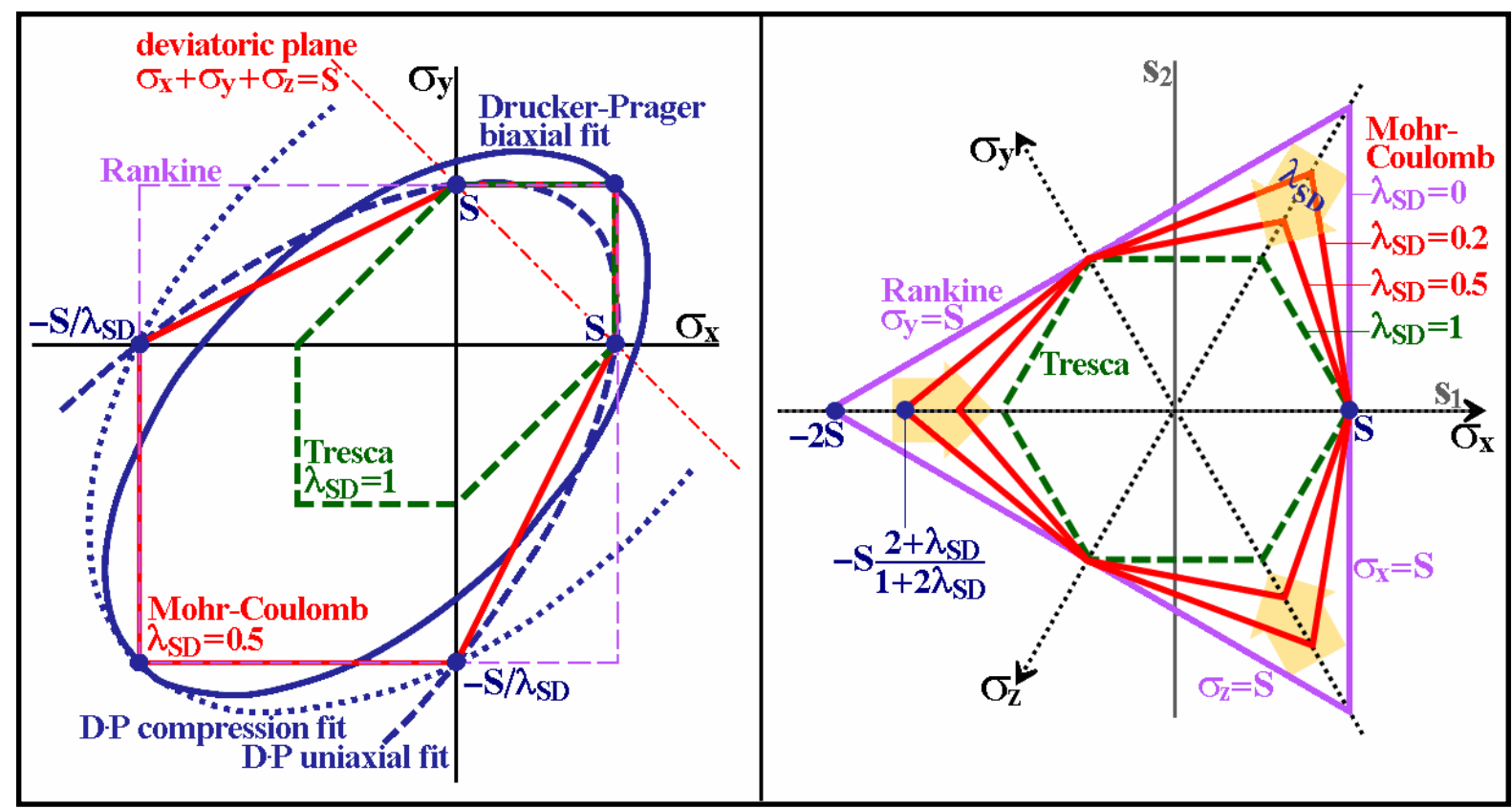

Figure 3. Cross sections of Tresca, Mohr-Coulomb (M-C), Rankine and Drucker-Prager (D-P) surfaces on the $\sigma_{z}=0$ plane, showing D-P uniaxial, biaxial and compression fits for $\lambda \mathrm{SD}=0.5$ (left), and cross sections on the tensile deviatoric plane $\sigma_{x}+\sigma_{y}+\sigma_{z}=S$ of Mohr-Coulomb's surfaces $\lambda \mathrm{SD}=\{0$, $0.2,0.5,1\}$ and their Tresca and Rankine limit cases (right), when all shear components are zero.

\subsection{Drucker-Prager}

The Mohr-Coulomb model can be interpreted as a generalization of Tresca for SD materials (with $\lambda \mathrm{SD} \neq 1$ ) to include mean stress effects through the normal component $\sigma_{\perp}$. In the same way, the Drucker-Prager surface has been proposed as a generalization of Mises, where the mean stress effect is included through the hydrostatic component $\sigma \mathrm{h}$, which is combined with the Mises stress in the yielding (or failure) criterion

$$
\underbrace{\sqrt{\left[\left(\sigma_{1}-\sigma_{2}\right)^{2}+\left(\sigma_{2}-\sigma_{3}\right)^{2}+\left(\sigma_{1}-\sigma_{3}\right)^{2}\right] / 2}}_{\sigma_{\text {Mises }}}+\alpha_{\mathrm{DP}} \cdot(\underbrace{\sigma_{1}+\sigma_{2}+\sigma_{3}}_{3 \sigma_{\mathrm{h}}})=\frac{2 \cdot \mathrm{S}}{1+\lambda_{\mathrm{SD}}} \equiv 2 \cdot \beta_{\mathrm{SD}}
$$

where $\beta \mathrm{SD}=\mathrm{S} /(1+\lambda \mathrm{SD})$ is the same effective shear strength from Mohr-Coulomb's model, with $S$ as either the yield or ultimate strengths, depending on how $\lambda$ SD was defined, and $\alpha D P$ is a material constant that calibrates the on influence. DruckerPrager's surface describes an infinite cone in the principal stress coordinates $\sigma_{1}-\sigma_{2}-$ $\sigma_{3}$, with vertex at the hydrostatic tensile state $\sigma_{x}=\sigma_{y}=\sigma_{z}=(2 / 3) \cdot \beta \mathrm{sD} / \alpha \mathrm{SD}$ and symmetry axis at the hydrostatic axis.

Depending on the $\alpha \mathrm{DP}$ calibration, the Drucker-Prager yield surface provides a smooth fit to Mohr-Coulomb's surface that intersects it in different number of vertices. If calibrated from $\alpha \mathrm{SD}$, i.e. $\alpha \mathrm{DP}=\alpha \mathrm{SD}=(1-\lambda \mathrm{SD}) /(1+\lambda \mathrm{SD})$, then Mohr-Coulomb is intersected in the principal axes for the uniaxial tension and compression strengths at

\footnotetext{
* Technical contribution to the $69^{\text {th }}$ ABM International Annual Congress and to the ENEMET, July $21^{\text {st }}-25^{\text {th }}$, 2014, São Paulo, SP, Brazil.
} 


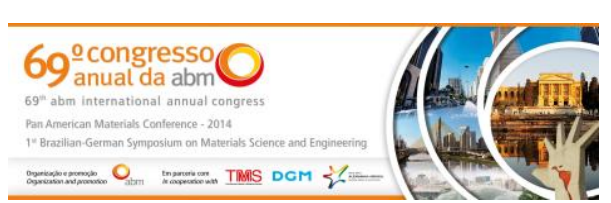

$S$ and $-S / \lambda s D$ (uniaxial fit), see Figure 3 . However, with this fit D-P is highly nonconservative for bi-axial compression. The compression fit obtained with $\alpha \mathrm{DP}=0$ provides a very good description of the uniaxial and bi-axial compression strengths $\sigma_{x}$ $=-\mathrm{S} / \lambda_{\mathrm{SD}}, \sigma_{\mathrm{y}}=-\mathrm{S} / \lambda_{\mathrm{SD}}$ and $\sigma_{\mathrm{x}}=\sigma_{\mathrm{y}}=-\mathrm{S} / \lambda_{\mathrm{SD}}$ (Figure 3 ), however it is highly nonconservative for bi-axial tension. A better approach is to fit Drucker-Prager's failure surface to both bi-axial tension and compression strengths, obtaining the bi-axial fit shown in Figure 3.

Another approach to accurately describe both tension and compression strengths is to use a composite yield surface, combining two or more yield surfaces. A good example involves grey cast iron, whose compression behavior is pressure-insensitive due to the closure of microcracks, but has a brittle tensile behavior that can be well decribed by Rankine's maximum principal stress theory. A good approximation of the yield surface of grey cast iron is thus the combination of Rankine's cubic surface in tension and Mises's cylindrical surface in compression, whose cross section on the $\sigma_{z}=0$ plane is shown in Figure 4. Note that Drucker-Prager's compression fit, obtained with $\alpha \mathrm{DP}=0$, is actually equivalent to the Mises pressure-insensitive surface for symmetrical tensile and compressive strengths $S / \lambda \mathrm{SD}$ and $-\mathrm{S} / \lambda \mathrm{sD}$.

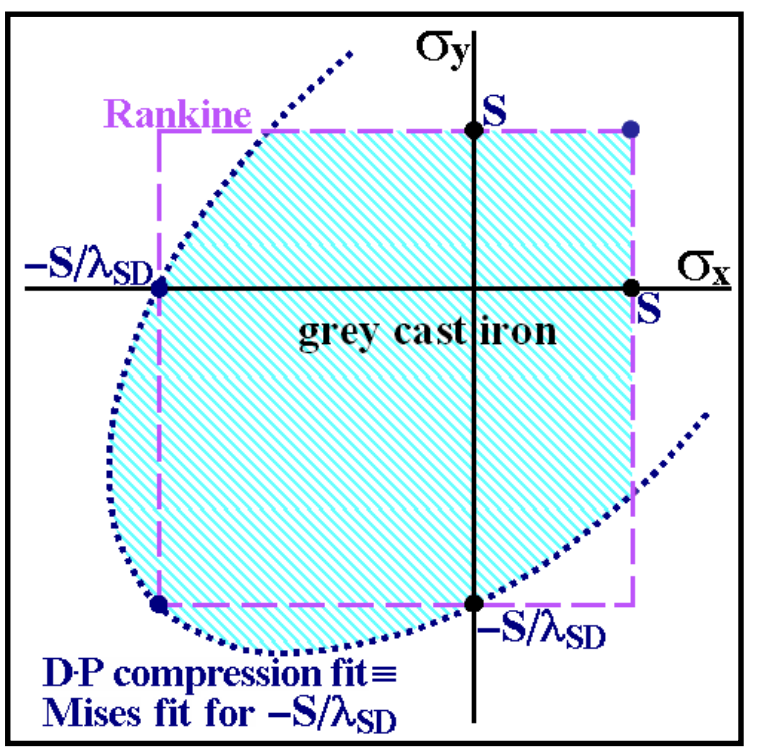

Figure 4. Cross section on the $\sigma_{\mathrm{z}}=0$ plane of the composite yield surface for grey cast iron, obtained from the combination of Rankine's and Mises' surfaces, assuming that it has $\lambda_{S D}=0.5$. Any combination of stresses in the shaded area would be considered elastic (or safe, in a failure analysis). The Mises surface can be obtained from Drucker-Prager's (D-P) compression fit.

\subsection{Gurson-Tvergaard-Needleman (GTN)}

The GTN model, proposed by Gurson, Tvergaard and Needleman [4,5], is a yield surface model that considers the effects of significant porosity caused by void coalescence in ductile fracture. Such porosity is quantified by the void volume fraction (VVF) $f$, which is defined as the ratio between the volume of all internal voids and the volume of the entire material, where $0 \leq f<1$. The yield surface equation becomes sensitive to the hydrostatic pressure $\sigma_{\mathrm{h}}$ through the use of a void volume function $\mathrm{f}^{\star}$, giving

\footnotetext{
* Technical contribution to the $69^{\text {th }}$ ABM International Annual Congress and to the ENEMET, July $21^{\text {st }}-25^{\text {th }}$, 2014, São Paulo, SP, Brazil.
} 


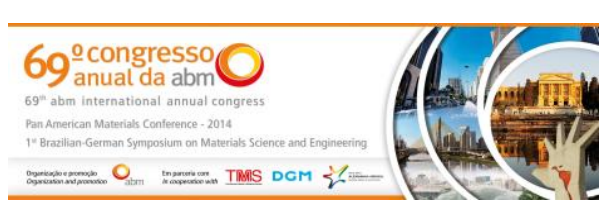

$$
\left(\frac{\sigma_{\text {Mises }}}{S}\right)^{2}+2 \cdot q_{1} \cdot f^{*} \cdot \cosh \left(q_{2} \frac{3 \sigma_{h} / 2}{S}\right)-\left(q_{3} \cdot f^{*}\right)^{2}=1
$$

where $q_{1}, q_{2}$ and $q_{3}$ are material constants, with typical $q_{1}=q_{3}=1.5$ and $q_{2}=1$, $\cosh (x)$ is the hyperbolic cosine function, and $S$ is a material strength e.g. $S=S Y$. This function $f^{*}$ acts as a damage parameter that is equal to $f$ from its initial $f=f_{0}$ for a virgin material until reaching a critical value $f=f_{c}$. For $f>f_{c}$, an accelerated void coalescence process requires a correction to be applied to $f^{\star}$, giving

$$
\mathbf{f}^{*}=\left\{\begin{array}{l}
\mathbf{f}, \text { if } \mathbf{f} \leq \mathbf{f}_{\mathbf{c}} \\
\mathbf{f}_{\mathbf{c}}+\frac{1 / \mathbf{q}_{\mathbf{1}}-\mathbf{f}_{\mathbf{c}}}{\mathbf{f}-\mathbf{f}_{\mathbf{c}}}, \text { if } \mathbf{f}_{\mathbf{c}}<\mathbf{f}<\mathbf{f}_{\mathbf{f}}
\end{array}\right.
$$

which is computed until $f$ reaches the fracture VVF $f_{f}$, when the material fails.

The GTN model requires the calibration of six parameters to predict the yield surface for a given $f$ : the three model parameters $q_{1}, q_{2}$ and $q_{3}$, which affect the shape of the yield surface; and the initial VVF $f_{0}$, critical VVF $f_{c}$ and fracture VVF $f_{f}$ (with $0 \leq f_{0}<f_{c}$ $<f_{f}<1$ ), obtained from a model of an incompressible material matrix with a void.

But the evolution of $f$ depends on additional equations that require the calibration of at least three other material parameters [6]. These parameters are used in differential equations to predict the increase in the VVF $f$ due to the nucleation of new voids as well as the growth of existing voids, which happen as the function of plastic straining. The GTN is the model of choice for ductile materials in crash simulations. It has also been successful in predicting the transverse plane strain fracture and the classic "cup-cone" ductile fracture, due to its explicit modeling of material voids. However, the calibration of the required nine material parameters is difficult because they are highly coupled. Also, the GTN model cannot accurately predict shear fracture, in special at low stress triaxiality, where void shearing is much more important than the void growth assumed in the model. Modifications to the GTN model to include the void shearing effect have been proposed in [6].

\section{MODEL COMPARISON}

A major difference between Mohr-Coulomb's and Drucker-Prager's models can be visualized in the example from Figure 5, which shows stress states under pure torsion in the 1-3 plane $\left(\sigma_{1}=-\sigma_{3}\right)$ combined with a principal stress $\sigma_{2}$ in compression (left) or tension (right). In both cases, the normal stress $\sigma_{\perp 13}=\left(\sigma_{1}+\sigma_{3}\right) / 2=0$ would not influence the opening or closure of a short crack on the critical plane 1-3, as predicted for a short crack by Mohr-Coulomb, resulting in an independence of $\sigma_{2}$. As a result, Mohr-Coulomb's model is often used for concrete and rock, where microcracks are usually unavoidable due to the material heterogeneity, materials for which the combination between the maximum shear stress $\tau_{\mathrm{ij}}$ and the normal stress $\sigma_{\perp \mathrm{ij}}$ that tends to open the microcrack faces sinergically contribute to material failure by fracture or even yielding.

\footnotetext{
* Technical contribution to the $69^{\text {th }}$ ABM International Annual Congress and to the ENEMET, July $21^{\text {st }}-25^{\text {th }}$, 2014, São Paulo, SP, Brazil.
} 


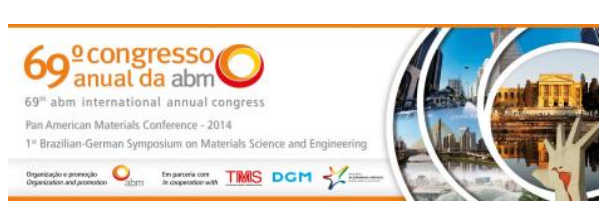

Drucker-Prager's use of invariants such as the Mises equivalent stress can be correlated with the invariant-based approaches used in multiaxial fatigue, such as the Sines' model [3]:

$$
\underbrace{\sigma_{\text {Mises }}+\alpha_{\mathrm{DP}} \cdot\left(3 \sigma_{\mathrm{h}}\right)=2 \cdot \beta_{\mathrm{SD}}}_{\text {Drucker-Prager }} \Rightarrow \underbrace{\Delta \tau_{\text {Mises }} / 2+\alpha_{\mathrm{S}} \cdot\left(3 \cdot \sigma_{\mathrm{hm}}\right)=\beta_{\mathrm{S}}}_{\text {Sines }}
$$

where $\alpha$ s and $\beta$ s are Sines' Stress Scale Factor (SSF) and shear fatigue limit, and $\Delta \sigma_{\text {Mises }}=\Delta \tau_{\text {Mises }} \sqrt{3}$. Sines' model can also be (and usually is) applied to metallic materials, even though Drucker-Prager was originally devised for granular materials. Therefore, such combination of Mises stress and hydrostatic stress $\sigma_{\mathrm{h}}$ is needed to predict failure of granular materials (e.g. using Drucker-Prager) due to the influence of $\sigma_{h}$ in particle cohesion, while it is also needed in metal fatigue predictions (e.g. using Sines) to account for the $\sigma_{h}$ influence on the opening or closure of microvoids or other microdefects initiated in multiple directions.

In summary, if the strength difference between tension and compression is caused by a directional phenomenon such as the opening and closure of microcracks (as in concrete), then Mohr-Coulomb's critical plane criterion should be adopted. Otherwise, if this difference is controlled by non-directional invariant quantities such as the hydrostatic stress and the Mises equivalent stress (as in granular materials), then Drucker-Prager's invariant criterion should be used instead. Note that for non$S D$ materials, where $\lambda S D \cong 1$ and $\alpha S D=\alpha D P \cong 0$, Drucker-Prager's and MohrCoulomb's criteria reduce to the Mises and Tresca models, respectively.

Mohr-Coulomb's infinite pyramid and Drucker-Prager's infinite cone are open surfaces in the compression direction, i.e. they assume that an infinite hydrostatic compression on would not cause yield or failure. However, for many geomaterials such as soils, a large enough hydrostatic compression $\sigma_{\mathrm{h}}<<0$ will induce permanent deformation. In these cases, an inferior limit to $\sigma$ can be imposed for each material to generate a closed (capped) yield surface, generating a finite pyramid or cone. This inferior limit may be assumed as a constant, or as a function of the Mises equivalent stress as in the Original and Modified Cam-Clay models [7], generating a curved capping surface. Another approach is to include a quadratic term of the hydrostatic stress $\sigma_{h}$ that will limit the size of the yield surface for both $\sigma_{h}<<0$ and $\sigma_{h}>>0$, as in Bresler-Pister's [8] extension of Drucker-Prager's criterion

$$
\sigma_{\text {Mises }}+\alpha_{\mathrm{BP}} \cdot(\underbrace{\sigma_{\mathrm{x}}+\sigma_{\mathrm{y}}+\sigma_{\mathrm{z}}}_{3 \sigma_{\mathrm{h}}})+\alpha_{\mathrm{BP}}^{\prime} \cdot(\underbrace{\sigma_{\mathrm{x}}+\sigma_{\mathrm{y}}+\sigma_{\mathrm{z}}}_{3 \sigma_{\mathrm{h}}})^{2}=2 \cdot \beta_{\mathrm{BP}}
$$

where $\alpha_{\mathrm{BP}}, \alpha_{\mathrm{BP}}^{\prime}$, and $\beta_{\mathrm{BP}}$ are Bresler-Pister's material constants that can be fitted, for instance, from measured values of the yield (or ultimate) strengths under uniaxial tension, uniaxial compression and biaxial compression. Originally devised for concrete, Bresler-Pister is also an excellent model to predict the yield behavior of polypropylene and polymeric foams, due to their quadratic pressure-dependence of the yield stress.

Although very popular in fracture simulations, the GTN model and its incremental plasticity formulation are not usually adopted in fatigue calculations, because its main advantage rests in the modeling of the critical region $f_{c}<f<f_{f}$, which is associated with a very small number of cycles in fatigue problems. In other words, the need to

\footnotetext{
* Technical contribution to the $69^{\text {th }}$ ABM International Annual Congress and to the ENEMET, July $21^{\text {st }}-25^{\text {th }}$, 2014, São Paulo, SP, Brazil.
} 


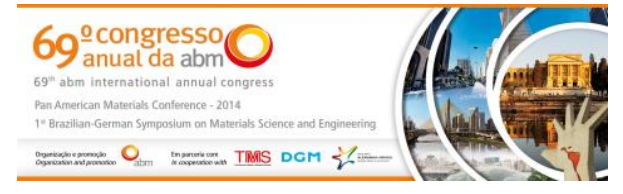

explicitly include void effects in a yield surface model is more associated with a ductile tearing problem near the end of the component's life rather than fatigue. The GTN model improvements would affect very little the number of cycles in fatigue crack growth predictions, with practically no effect in crack initiation calculations in special in the high-cycle regime.

\section{CONCLUSIONS}

This work reviewed and compared the main yield surface equations for isotropic materials. Even though the yield behavior of most metallic materials can be modeled using Mises and Tresca surfaces, the consideration of the hydrostatic stress or the normal stress acting on a critical plane can be useful for pressure-sensitive materials or even to predict the fatigue behavior of both ductile and brittle materials, as seen in the analogies from Equations (13-14).

It was shown that Mohr-Coulomb's pressure-sensitive model is a good choice for concrete and rock, where the failure mechanism involves microcracks under the combined effects of normal stresses that tend to open their faces and shear stresses that tend to propagate them. Such model can be extended to multiaxial fatigue calculations using a critical plane approach, generating the Mohr-type multiaxial damage models such as Findley's, applicable even to pressure-insensitive materials such as most metals.

It was also shown that Drucker-Prager's pressure-sensitive model is more appropriate for soils and granular materials, due to the use of invariants such as the Mises equivalent stress. Such model can be extended to multiaxial fatigue calculations using an invariant-based approach, generating e.g. the Sines model, applicable as well to metals and several other non-granular materials.

Finally, it was concluded that the GTN model is not needed in yielding or fatigue calculations, since its main application is to model fracture by ductile tearing and void coalescence.

\section{REFERENCES}

1 Valanis KC. A theory of viscoplasticity without a yield surface. Archiwum Mechaniki Stosowanej (Archive of Mechanics). 1971;23(4):517-551.

2 Meggiolaro MA, Castro JTP. An Improved Multiaxial Rainflow Algorithm for NonProportional Stress or Strain Histories - Part I: Enclosing Surface Methods. International Journal of Fatigue. 2012;42:217-226.

3 Socie DF, Marquis GB. Multiaxial Fatigue. SAE 1999.

4 Gurson AL. Continuum theory of ductile rupture by void nucleation and growth: Part I Yield criteria and flow rules for porous ductile media. ASME Journal of Engineering Materials and Technology, ASME. 1977;99:2-15.

5 Tvergaard V, Needleman A. Analysis of the cup-cone fracture in a round tensile bar. Acta Metallurgica, 1984;32:157-169.

6 Xue L. Constitutive modeling of void shearing effect in ductile fracture of porous materials. Engineering Fracture Mechanics. 2008;75:3343-3366.

7 Roscoe $\mathrm{KH}$, Burland JB. On the generalised stress-strain behaviour of wet clay. In Engineering Plasticity, Cambridge University Press, 1968:535-609.

8 Bresler B, Pister KS. Strength of concrete under combined stresses. ACI Journal. 1958;551(9):321-345.

\footnotetext{
* Technical contribution to the $69^{\text {th }} A B M$ International Annual Congress and to the ENEMET, July $21^{\text {st }}-25^{\text {th }}$, 2014, São Paulo, SP, Brazil.
} 